\title{
Minilaparoscopic total hysterectomy
}

\begin{abstract}
Hysterectomy is one of the most common gynecological procedures in the world. Duringthe 1990s it was widely recognized that the superior patient benefits of laparoscopic surgery could be attributed in large part to the reduction in operative trauma, mediated by smaller accessincisions, gentle tissue handling, and decreased need of retraction and dissection. Mini-laparoscopic surgery has come forward a new technique especially in recent years. The concept behind minilaparoscopy is thats maller instruments cause less abdominal wall trauma and thus reduce in cision related morbidity and minimize pain and the stress response to surgery.
\end{abstract}

Keywords: minilaparoscopy; gynecology; hysterectomy
Volume 2 Issue 6 - 2016

\author{
Servet Gencdal,' Emre Ekmekci, ${ }^{2}$ Hüseyin \\ Aydogmuș,' Serpil Aydogmuș, ${ }^{2}$ Sefa Kelekci ${ }^{2}$ \\ 'Department of Obstetrics and Gynecology Clinic, Izmir \\ Atatürk Education and Research Hospital,Turkey \\ ${ }^{2}$ Department of Obstetrics andGynecology, Izmir Katip Celebi \\ University, Turkey
}

Correspondence: Servet Gençdal, Department of Obstetrics and Gynecology Clinic, Izmir Atatürk Education and Research Hospital, Ministry of Health, Izmir, Turkey, Tel 505 3|43 I36, Fax +900232 2431530, Email servetgencdal@hotmail.com

Received:September II, 2016 | Published: October 19, 2016

\section{Introduction}

Hysterectomy is the most commonly performed major gynecologic operation and it is reported that, 430.000 operations have been performed in USA in 2010 year. ${ }^{1}$ In USA, methods for hysterectomies that are benign gynecologic conditions indicated were, $56 \%$ abdominal, $20 \%$ were laparoscopic, $19 \%$ were vaginal and $5 \%$ were roboticin the year $2009 .^{2}$ Laparoscopy is a surgical procedure that has been used widely in medicine over 30 years. The faster recovery time, the minimizing of pain, hospitalization and the better aesthetic result are some of the advantages which made laparoscopy very popular among patients and surgeons. But initially its use in gynecology was restricted to the diagnosis of infertility and sterilization procedures. Gradually with time and increasing expertise it is being used as the diagnostic as well as the therapeutic modality in different gynecological problems. ${ }^{3-5}$ Laparoscopic hysterectomy is the first time defined in 1989 by Reich. ${ }^{6}$ Nowadays, especially in hysterec to my operations due to benign causes, a transition from open surgery to laparoscopic surgery is noticable. Advances in instrumentation and progress in fiber optic technology have armed the surgeons with smaller caliber instruments and better optics and light sources, thus triggering the emergence of needle scopic or minilaparoscopic surgery. There is no consensus in the literature about the strict definition of "minilaparoscopy," although several reports have suggested that the word be reserved only for laparoscopic operations performed entirely using 3-mm trocars with the only possible exception of the umbilical port. ${ }^{7}$ Minilaparoscopic surgery had been introduced several years ago, but it was initially reserved for exclusively diagnostic procedures,${ }^{8}$ whereas interventional use of 3-mm instruments was mainly considered as a mere complementary addition to standard 5-10-mm trocars. The concept behind minilaparoscopy is that smaller instruments cause less abdominal wall trauma and thus reduce incision related morbidity and minimize pain and the stress response to surgery Nevertheless, the procedure had not been applied in adult surgery until recently. The main reason for the latter observation was that initial mini-laparoscopic instruments showed some limitations; suboptimal vision, loose grasping, defective irrigation or suction and decreased instrument durability were the main problems associated with needle scopic equipment. Second generation mini-laparoscopic instruments have addressed the former drawbacks and currently, a wide range of needle scopic instruments have been added in the armamentarium of laparoscopic surgeons. ${ }^{9}$ In the field of gynecologic surgery, the first operative applications regarded adnexal procedures ${ }^{10}$ andhysterectomies, ${ }^{7}$ One of the main contributors to these advancements has been the introduction of efficient bipolar coagulation that can warrant adequate hemostatic control of moderatecaliber blood vessels, as in the case of the uterine pedicles. Today, the use of mini-laparoscopic instruments in endoscopic surgery is becoming increasingly common. The introduction of $3-\mathrm{mm}$ trocars for laparoscopic hysterectomy procedures has provento be as effective as standard-caliberaccesses. ${ }^{7,11}$

\section{Hysterectomy undications}

The most common hysterectomy indications are, uterine leiomyomas, adenomyosis, idiopathic a normal uterine bleeding, endometriosis and uterine prolapsus. Also, hysterectomy is being performed due to malignant processes like uterine, ovarian and cervical malignancies.

\section{Minimally required ınstruments}

Instruments required for mini-laparoscopic hysterectomy: monitor, camera and camera system unit, light source and insfluation system, laparoscops (3 and 5mm diameter, 0 and 30degrees Karl Storz, Germany), verres needle(14G width, $12-15 \mathrm{~cm}$ length), $3 \mathrm{~mm}$ diameter trocar, grasping, dissection/cutting, a suction-washing system and monopolar or bipolar electro surgical instruments (Gyrus Medical Inc., Minneapolis, MN) and uterine manipulator slike RUMI uterine manipulator (Cooper-surgical), V Care uterine manipulator/elevator (conmedendo surgery).

\section{Preoperative evaluation and preparation}

Preoperatively, treatment alternatives and operative risks should be discussed with patient prior to mini laparoscopic total hysterectomy and informed consent should be taken. Preoperative tests should involve medical, surgical, gynecologic and obstetrical history. In case of suspected gynecological malignancy, cervical smear and endometrial sampling should be taken. Antibiotic prophylaxis should 
be given to all patients prior to surgical procedures, mechanical or pharmacological thrombo prophylaxis for venous thrombo embolism should be implemented, but mechanical bowel cleaning is not recommended before surgery. ${ }^{12}$

\section{Operative technique}

Small intestine and momentum should be examined carefully about possible verres and trocar damage. In case of pelvic or intra abdominal adhesion, adhesiolysis should be completed for better visualization of pelvic structures (ureter, vessels) and restoration of normal pelvic anatomy. It is important to define bilateral ureters before hysterectomy. Round ligament is being cauterized and cut by a bipolar PK System MoLly Forceps (Gyrus Medical Inc., Minneapolis, MN) instrument, anterior and posterior peritoneal leaves of broad ligament are dropped and separated (to aid mobility especially for wide and fixes uterus). The type of adnexal surgery is depended to presence of ovarian preservation. If ovaries and tubas will be preserved, utero-ovarian ligaments are cauterized and cut by an electrosurgical instrument. If salping ooopherectomy will be applied, in fund ibulopelvic ligaments are cauterized and cut. Before this process, ureters should be defined transperitoneally or retroperitoneally by dissection to avoid ureter damage. Anterior leaf of broad ligament is being cut till to the vesicouterine peritoneal fold for this. Uterine arteries are defined and skelitized after dissection of posterior broad ligament. After definition of both ureters, bilateral uterine arteries are cauterized and cut by electrosurgical instruments on internal cervical os level. During this process, upward traction of the uterus by a manipulator produces ensuring the ureter away from the electrosurgical tool is very useful in terms of minimizing the damage to ureter. After dissection of parametrial tissues, colpotomy stage starts. A circular incision around cervix is made by a $3 \mathrm{~mm}$ diameter monopolar hook. Cervical capped uterine manipulators both simplify the process and reduces the damage risk to ureters. Extreme thermal damage should be avoided during colpotomy for quick healing at vaginal cuff. Uterus is taken outside trans vaginally. Vaginal cuff can be closed by various suture techniques related to surgeon's experience. Some surgeons close cuff trans vaginally. In a retrospective study, comparing vaginal cuff closure transvaginal or laparoscopic during total laparoscopic hysterectomy, it is reported that transvaginal cuff closure reduces vaginal cuff dehiscence. ${ }^{13,14}$ After vaginal cuff closure, surgical area is re-examined for hemostasis under low intraperitoneal pressure.

\section{Complications}

Complications and their management of total laparoscopic hysterectomy and abdominal hysterectomy are similar. In large studies, complication ratios of laparoscopic hysterectomy are reported as; transfer to laparatomy $(2,7-3,9 \%)$, hemorrhage $(2-5,1 \%)$, urinary tractinjury $(1,2-3 \%)$, vaginal cuff dehiscence (1-2\%), intestinalinjury $(0,2-0,4 \%) .^{15,16}$ Complication rates are reported similar between mini-laparoscopy and conventional laparoscopy in large studies but postoperative pain and analgesic require are reported lower in minilaparoscop. ${ }^{17}$ Hernia formation in trocar region: When compared to laparatomic surgery, one of the most significant advantages of laparoscopy is to enable decrease in postoperative hernia formation. The incidence is between 0.06 and $1 \%$ andthis rate is approximately 10-100times lower when compared with laparotomy. ${ }^{18}$ By using mini-laparoscopy also it can be possible to reduce postoperative hernia formation, subcutaneous or sub facial bleeding and hematoma formation. ${ }^{19}$

\section{Conclusion}

Recently, wide use of total laparoscopic hysterectomy in plenty of centers has forced gynecologists to learn this surgery. The advantages and comfort of total mini laparoscopic hysterectomy and awareness about these by patients and surgeons will make it more popular in future. However, because long learning curve in this surgery, adaptation to surgical instruments initially is hard and complexity of management of complications laparoscopically, starting mini laparoscopy with less complicated cases initially and then with advancing experience to start total laparoscopic hysterectomy will improve operative success and reduce complications.

\section{Acknowledgements}

None.

\section{Conflict of interest}

The author declares no conflict of interest.

\section{References}

1. Wright JD, Herzog TJ, Tsui J, et al. Nation wide trends in the performance of in patient hysterectomy in the united states. Obstet Gynecol. 2013;122(2 Pt 1):233-241.

2. Cohen SL, Vitonis AF, Einarsson JI. Updated hysterectomy surveillance: Factors associated with minimally invasive hysterectomy, A cross-sectionalanalysis. 2014;18(3):e2014.00096.

3. American College of Obstetricians and Gynecologists, Operative Laparoscopy, ACOG Educational Bulletinno. 239, American College of Obstetricians and Gynecologists, Washington, USA: DC; 1997.

4. Darzi A, Mackay S. Recentadvances in minimal Access surgery. BMJ. 2002;324( 7328):31-34.

5. Pittaway DE, Takacs P, Bauguess P. Laparoscopic and exectomy: acomparison with laparotomy. Am J Obstet Gynecol. 1994;171(2):385-391.

6. Reich H. Laparoscopic hysterectomy. Surg Laparosc Endosc. 1992;2(1):213-216.

7. Ghezzi F, Cromi A, Siesto G, et al. Minilaparoscopic versus conventional laparoscopic hysterectomy: results of a randomizedtrial. J Minim Invasive Gynecol. 2011;18(4):455-461.

8. Bruhat MA, Goldchmit R. Minilaparoscopy in gynecology. Eur J Obstet Gynecol Reprod Biol. 1998;76(2):207-210.

9. Krpata DM, Ponsky IA. Needle scopic surgery: what's in the toolbox? Surg Endosc. 2013;27(3):1040-1044.

10. Ghezzi F, Cromi A, Colombo G, et al. Minimizing ancillary ports size in gynecologic laparoscopy: A randomized trial. J Minim Invasive Gynecol. 2005;12(6):480-485.

11. Ghezzi F, Cromi A, Siesto G, et al. Needle scopichysterectomy: Incorporation of 3-mm instruments in total laparoscopic hysterectomy. Surg Endosc. 2008;22(10):2153-2157.

12. Siedhoff MT, Clark LH, Hobbs KA, et al. Mechanical bowel preparation before laparoscopic hysterectomy: a randomized controlled trial. Obstet Gynecol. 2014;123(3):562-567.

13. Whiteside JL, Barber MD, Walters MD, et al. Anatomy of ilioinguinal and iliohypogastric nerves in relation to trocar placement and low transverse incisions. Am J Obstet Gynecol. 2003;189(6):1574-1578.

14. Uccella S, Ceccaroni M, Cromi A, et al. Vaginal cuffdehi scence in a series of 12,398 hysterectomies: effect of different types of colpotomy and vaginal closure. Obstet Gynecol. 2012;120(3):516-523. 
15. Canis $\mathrm{M}$, Botchorishvili $\mathrm{R}$, Ang $\mathrm{C}$. When is laparo to my needed in hysterectomy for benign uterine disease? J Minim Invasive Gynecol. 2008; $15: 38$.

16. Hur HC, Donnellan N, Mansuria S, et al. Vaginal cuffdehiscence after different modes of hysterectomy. Obstet Gynecol. 2011;118(4):794-801.

17. Fanfani F, Fagotti A, Rossitto C, et al. Laparoscopic, minilaparoscopic and single porthysterectomy: perioperative outcomes. Surg Endosc. 2012;26(12):3592-3596
18. Chapron C, Pierre F, Harchaoui Y, et al. Gastro intestinal injuries during gynaecological laparoscopy. Hum Reprod. 1999;14(2):333-337.

19. Nomura H, Okuda K, Saito N, et al. Mini-laparoscopic surgery versus conventional laparoscopic surgery for patients with endometriosis. $G y$ necology and Minimally Invasive Therapy. 2013;2(3):85-88. 